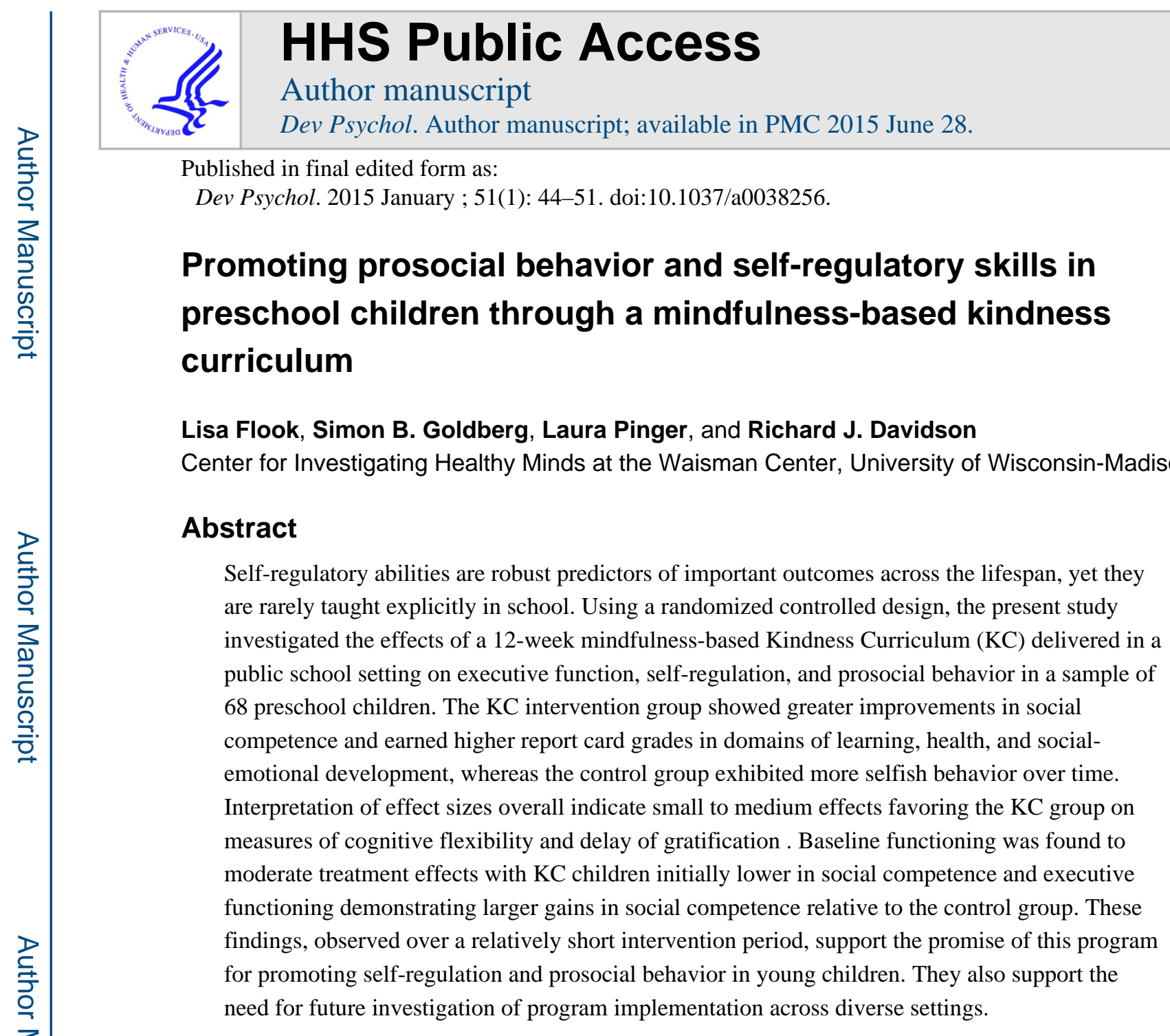

Keywords

mindfulness; executive function; prosocial behavior; preschool; early childhood

Social, emotional, and cognitive functioning are deeply intertwined throughout the lifespan. As such, self-regulatory skills are increasingly recognized as important contributors to school success. The capacity to regulate attention and emotion are forms of self-regulation that provide a foundation for school readiness by supporting dispositions conducive to learning and maintaining positive social relationships (Blair, 2002). The importance of developing such competencies early in life is underscored by longitudinal research demonstrating that self-regulation in childhood predicts health, financial stability, and educational attainment into adulthood (Moffitt et al., 2011). There is particular interest in training during early childhood given the malleability and plasticity associated with this period of development. Furthermore, economists have demonstrated that investments in early childhood education pay for themselves, yielding a return of $7 \%$ or more (Heckman, 2011). Investment in early education, therefore, has the potential to increase health and reduce risk behaviors over the lifespan, thus reducing overall societal costs. 
Healthy functioning across academic and social contexts requires exercising self-regulatory ability in the pursuit of short and long-term goals. Self-regulation involves modulating feelings, thoughts, and behavior, and is associated with academic achievement and social competence, both concurrently and prospectively (Eisenberg, Spinrad, \& Smith, 2004; Spinrad et al., 2006). In a classic delay of gratification paradigm, self-regulatory ability at age 4 (indexed by waiting a longer time for a reward) predicts attentional capacity, selfcontrol and frustration tolerance years later during adolescence (Eigsti et al., 2006; Mischel et al., 1988; Shoda et al., 1990). Further, children who are better able to delay also score higher on the SAT and are perceived as more interpersonally competent by parents and peers (Mischel et al., 1989). In contrast, deficits in self-regulation can significantly interfere with learning (Barkley, 2001). Thus, the ability to enhance and strengthen attention and emotion regulatory resources warrants further investigation.

Attention and executive functioning play a key role in effective self-regulation. Executive functions (EFs) refer to an array of related, yet distinct cognitive processes, such as cognitive flexibility, inhibitory control, and working memory, which impact all areas of functioning (Anderson, 2002; McCloskey, Perkins, \& Van Divner, 2009; Miyake et al., 2000; Zelazo, Carlson, \& Kesek, 2008). The development of prefrontal cortical (PFC) regions are linked to attention and EFs, abilities which are present in a rudimentary form at the beginning of life and undergo rapid development congruent with brain growth during the childhood years (Diamond, 2002). EFs are a fundamental component of school success and predict academic performance above and beyond general levels of intelligence (Blair \& Razza, 2007). Therefore, early childhood is an opportune period of development for training such skills.

Despite evidence highlighting the importance of such self-regulatory skills to academic and life success, these skills are not often explicitly taught in school. Rather, instruction tends to emphasize academic knowledge and performance on standardized tests. There has been a surge in interest among educators, parents and policy makers in addressing children's social and emotional development in addition to academic skills, but as yet no clear consensus exists on what constitute the best strategies and methods for cultivating these positive qualities in young children (Greenberg et al., 2003). Emerging scientific evidence supports the training of these skills in children through a variety of modalities including mindfulnessbased practices (Diamond \& Lee, 2011).

\section{Mindfulness Training and Education}

Mindfulness training enhances attention and EF by bringing awareness to a particular attentional object, whether it is the breath, external stimuli, thoughts or emotions. It entails noticing when the mind has wandered from its object of attention (monitoring) and returning attention back to the chosen object (shifting/cognitive flexibility). Training increases the ability to maintain engagement of self-regulatory neural circuits (PFC) resulting in improved sustained attention and emotion regulation (Lutz, Slatger, Dunne, \& Davidson, 2008; MacLean et al., 2010). 
Through the training of attention, the qualities of kindness and care towards oneself and others can be cultivated implicitly and explicitly. Young children, for instance, engage in setting daily intentions and age-appropriate practices to extend care and well wishes. A similar form of mental training is associated with increased activity in cortical areas responsible for empathy and compassion among adults (Lutz, Brefczynski-Lewis, Johnston, \& Davidson, 2008) with measurable change in altruistic behavior and corresponding neural activity in as few as two weeks (Weng et al., 2013). Thus, these practices may effect changes induced through neural plasticity that support prosocial behavior and academic success (Davidson et al., 2012; Davidson \& McEwen, 2012).

While the popularity of mindfulness programs for children has increased rapidly, there is a paucity of empirical research on the effects of such practices. Initial studies of mindfulness training with children show promising effects (e.g., Flook et al., 2010; Napoli, Krech, \& Holley, 2005) and a review of meditation-based interventions among youth reported a median effect size between .27 to .70 for psychological and behavioral outcomes (Black, Milam \& Sussman, 2009). With some exceptions, existing research is primarily limited by a lack of randomized controlled trials and reliance on questionnaire measures. The present study uses a randomized controlled design to assess the effects of a mindfulness-based prosocial skills training curriculum on a range of cognitive and behavioral outcomes in preschool children. In addition, we examine moderators of the intervention effect with the hypothesis that those who are lower in prosocial skills and EF as assessed behaviorally at baseline will improve most in terms of teacher ratings of their social-competence over time.

\section{Methods}

\section{Participants}

Seven classrooms were recruited from six different elementary schools within a public school district in a medium-sized Midwestern city. Within these schools, $37.9 \%$ of children are considered socioeconomically disadvantaged. A total of 99 children were invited to participate and parents of 68 indicated they wished to enroll their child in the study (acceptance rate $=68.7 \%)$. The sample included 40 Caucasian $(58.8 \%), 8$ Hispanic $(11.8$ $\%), 4$ African American (5.9\%), 7 Asian/Pacific Islander (10.3\%), 8 “Other"/mixed ethnicity children (11.8\%), 34 girls (50.0\%) and 33 boys (48.5\%), with a mean age of 4.67 years $(S D=.27)$. Of the parents, $49(72.1 \%)$ were four-year college graduates and 18 (26.5 $\%$ ) were not (demographic data were missing for one parent and child).

Participants were randomly assigned by classroom to either a mindfulness-based "Kindness Curriculum" (KC) intervention or a wait-list control group ( $n=30$ from three classrooms and 38 from four classrooms, for $\mathrm{KC}$ and control group respectively). Participants were assessed in individual testing sessions before and after the training period. All procedures for this study were approved by both the university and school district research review boards. 


\section{Kindness Curriculum (KC) Intervention}

The intervention group received a 12-week mindfulness-based prosocial skills training designed for preschool-age children (see Appendix for sample lessons). The foundation of the $\mathrm{KC}$ is mindfulness practice, aimed at cultivating attention and emotion regulation, with a shared emphasis on kindness practices (e.g., empathy, gratitude, sharing). The preschool KC is progressive in nature and incorporates children's literature, music, and movement to teach and stabilize concepts related to kindness and compassion. The curriculum was taught by experienced mindfulness instructors in a secular manner and was provided universally to children as part of their standard classroom instruction during regular school hours. Student training in the KC consisted of two 20-30 minute lessons each week over a 12-week period, totaling approximately 10 hours of training.

\section{Measures}

Teacher-rated social competence (TSC)—Teacher's ratings of their student's social competence were obtained using items from the Teacher Social Competence Scale (Conduct Problems Prevention Research Group, 1995). The measure (overall $a=.94$ ) was comprised of two subscales: a prosocial behavior subscale ( 7 items, showing empathy and compassion for others' feelings, $a=.95$ ) and an emotion regulation subscale (5 items, e.g., stopping and calming down when excited or upset, $a=.82$ ). Items were rated on a 6-point Likert-type scale with higher scores indicating greater social competence.

Sharing task-This task was designed for use in the current study and consisted of four separate trials in which children distributed stickers between themselves and a target recipient. The four target recipients included a most and least liked peer (identified by the participant) from their class, an unfamiliar child, and a child who was sick. In each of the four trials, children were presented with an envelope for themselves labeled "me" and an envelope with a picture of the designated target recipient.

Children were given 10 stickers at the beginning of each trial and told they could keep as many as they would like for themselves and give as many as they would like to the other person. Scores were computed for each trial along with a total score average score that reflected the number of stickers put in the "me" envelope across all four trials.

Delay of gratification-The delay of gratification task was based on Prencipe and Zelazo's (2005) procedure. The task included nine test trials requiring a choice between having a smaller reward "now" ( 1 item) or a larger reward "later" (2, 3 or 5 items). In the present study, rewards included food, crayons, and tokens (which could be exchanged for a variety of small toys). Scores were computed for all trials and each contingency representing the mean number of times the child chose the delay condition.

Dimensional change card sort task (DCCS) - A computerized version of the dimensional change card sort (DCCS) task was used to assess cognitive flexibility, a core aspect of executive function (Garon, Bryson, \& Smith, 2008). This task was taken from the National Institute of Health (NIH) Toolbox Cognitive Function Battery (Zelazo et al., 2013). 
The task requires participants to sort bivalent test cards first by one dimension (e.g., shape) and then sort the same cards by a second dimension (e.g., color).

The DCCS consists of three test blocks - pre-switch, post-switch, and mixed. Practice trials (four trials for each dimension), are followed by the pre-switch block (five trials). The postswitch block (five trials) requires sorting by the second dimension. The mixed block (40 trials) includes shifting between sorting dimensions.

Scoring followed recommended procedures (Zelazo et al., 2013) including the computation of a composite score that allows an equal contribution for accuracy and reaction time. Composite scores were calculated for all trials as well as for only post-switch trials. Although the scoring procedures recommend using only the mixed block, the post-switch block was used given the large percentage of participants not receiving the mixed block due to low accuracy (the task ends if accuracy cut-off is not met). This post-switch block was seen as the next best proxy for cognitive flexibility as it still requires a shift in sorting dimension.

Flanker task-A computerized flanker task, also from the NIH Toolbox Cognitive Function Battery (Zelazo et al., 2013), was used to measure inhibitory control, another core aspect of executive function (Garon et al., 2008). In this task, participants were presented with a row of five stimuli (either fish or arrows) and pressed one of two buttons indicating the direction the middle stimulus (either a fish or arrow) is pointing. During congruent trials all the stimuli are pointing the same direction while in the incongruent trials the flanking stimuli are pointing the opposite direction from the middle stimulus.

The Flanker task included three blocks: practice (four trials), fish (20 trials), and arrows (20 trials). Scoring followed recommended procedures (Zelazo et al., 2013) including the computation of a composite score that reflects equal contributions of accuracy and reaction time.

Both the Flanker and DCCS tasks have demonstrated excellent test-retest reliability (ICC $=$. 92 for both tasks) as well as good convergent and discriminant validity (Zelazo et al., 2013).

School grades-Children's grades reflecting performance for the second half of the academic year were obtained from school records. Teachers assigned grades in five different domains using a 4-point scale with higher scores indicating the child met or exceeded expectations: Approaches to Learning ( 3 items, persists with self-initiated activities, $\alpha=$. 76), Cognition and General Knowledge (7 items, sorts and/or describes objects by size, shape, color, or use, $a=.72$ ), Health and Physical Development (5 items, demonstrates balance and strength, $\alpha=.77$ ), Language Development and Communication ( 9 items, retells a familiar story in sequence, $a=.87$ ), Social and Emotional Development ( 7 items, displays age appropriate self-control, $a=.86$ ).

\section{Data Analysis}

Independent $t$-tests were conducted to compare groups at baseline. Repeated measures analysis of variance (RMANOVA) analyses were conducted with baseline scores entered as 
covariates to assess for group by time interactions. Subsequent RMANOVA models also included child gender and age as covariates. Paired $t$-tests were used post hoc to examine change from pre- to post-test within intervention and control groups separatelyin cases where the omnibus F-value from the RMANOVAs showed a significant effect (i.e., a significant group by time interaction effect controlling for baseline levels). For data only reported at post-test (i.e., school grades), independent t-tests were used comparing groups post-intervention. In addition, given the exploratory nature of this study and the relatively small sample size, we conducted both formal significance testing (using RMANOVAs and $t$ tests) as well as generated effect sizes reflecting group differences. For measures assessed at both pre- and post-, we computed both within-group pre-post effect sizes (Cohen's $d$, using pooled pre- and post-test standard deviations) and between-group effect sizes (computed as the difference between within-group $d \mathrm{~s}$, with $\mathrm{KC} d$ minus control group $d$, equivalent to Becker's [1988] del). For outcomes assessed only at post-test (school grades), only a between-group $d$ was computed, also using a pooled standard deviation. Effect sizes were interpreted based on Cohen's (1988) guidelines.

In order to assess for individual differences in response to intervention, ANOVA models were constructed examining baseline by group interactions as a predictor of change. The small number of level two units (i.e., classrooms) precluded the possibility of modeling treatment effects in multilevel models (Snijders \& Boskers). Intraclass correlation coefficients (ICCs) were computed to index the amount of variance in outcomes attributable to classrooms.

\section{Results}

There were no significant differences at baseline on any demographic variables or other measures assessed at baseline (i.e., delay of gratification, sharing task, TSC, DCCS, Flanker; $p>.05)$.

\section{Teacher-rated social competence (TSC)}

A significant group by time interaction was found in RMANOVA analyses (controlling for baseline levels) for TSC total score $(F[1,63]=6.78, p=.011)$ as well as the prosocial behavior $(F[1,63]=4.37, p=.041)$ and emotion regulation subscales $(F[1,63]=10.12, p$ $=.002)$. Results from all RMANOVA analyses along with group means and SDs are reported in Table 1. Significant pre-post change with large effect sizes was found for both groups in post hoc paired $t$-tests for TSC total score (KC: $t[28]=8.06, p<.001, d=1.31$; control: $t[36]=8.79, p<.001, d=1.05)$ as well as prosocial behavior (KC: $t[28]=8.03, p$ $<.001, d=1.27$; control: $t[36]=8.51, p<.001, d=0.98)$ and emotion regulation subscales (KC: $t[28]=6.81, p<.001, d=1.22$; control: $t[36]=6.25, p<.001, d=0.97$ ). A small between-group $d$ (computed as the difference between within-group $d$ s) was found favoring the KC group for TSC total score $(d=0.26)$ as well as for the prosocial behavior $(d=0.29)$ and emotion regulation subscales $(d=0.25)$. RMANOVA analyses conducted post hoc including gender and age did not change significance tests for the TSC total score or emotion regulation subscales; the RMANOVA including prosocial behavior was no longer significant $(F[1,60]=2.71, p=.105)$. 


\section{Sharing task}

A significant group by time interaction was found with the RMANOVA analysis for sharing (total stickers kept for self), indicating that the control group kept significantly more for themselves over time relative to the $\mathrm{KC}$ group $(F[1,58]=6.53, p=.013$; Fig. 1$)$. This result remained significant when controlling for gender and age. Paired $t$-tests conducted post hoc revealed a significant increase in stickers kept for self in the control group $(t[33]=2.28, p$ $=.029, d=0.48)$ but not in the $\mathrm{KC}$ group $(t[26]=0.51, p=.613, d=0.15)$. The betweengroup effect $(d=-0.33)$ was small.

\section{Delay of gratification}

No significant RMANOVA analyses were noted for the delay of gratification task (all trials: $F[1,59]=1.39, p=.244$, one vs. two: $F[1,59]=0.02, p=.901$, one vs. three: $F[1,59]=$ $1.93, p=.170$, one vs. five: $F[1,59]=2.47, p=.121)$. Within-group $d$ s reflected small to medium sized increases in the KC group $(d \mathrm{~s}=0.42,0.16,0.47$, and 0.48 for all trials, one vs. two, one vs. three, and one vs. five respectively) and small increases in the control group ( $d \mathrm{~s}$ $=0.19,0.20,0.18$, and 0.11 for all trials, one vs. two, one vs. three, and one vs. five respectively). Examining between-group $d \mathrm{~s}$, a small effect size was noted across all trials ( $d$ $=0.23)$, in one vs. three $(d=0.29)$, and in one vs. five $(d=0.37)$ trial types in the direction of more delay in the $\mathrm{KC}$ group relative to the control group. A very small effect size ( $d=$ -0.04 ) was noted favoring the control group on one vs. two trials.

\section{Dimensional change card sort task (DCCS)}

No significant RMANOVA analyses were noted for the DCCS task (all trials: $F[1,53]=$ $0.08, p=.785$, post-switch trials: $F[1,41]=1.54, p=.222$ ). Medium to large effects were found pre-post for both the $\mathrm{KC}$ group $(d \mathrm{~s}=0.57$ and 0.87 , for all trials and post-switch respectively) and control group ( $d s=0.70$ and 0.44 , for all trials and post-switch respectively). Examining between-group $d$ s, a small to medium effect size was noted in post-switch trials $(d=0.43)$ in the direction of improved cognitive flexibility in the KC group relative to the control group. A very small effect size favoring the control group was noted across all trials $(d=-0.13)$.

\section{Flanker task}

The RMANOVA analysis was not significant for the Flanker task $(F[1,56]=0.62, p=$. 434). The pre-post within-group effect size was medium sized in both groups ( $d \mathrm{~s}=0.47$ and 0.64 , for $\mathrm{KC}$ and control groups respectively). The between-group effect size was small in magnitude $(d=-0.17)$ in the direction of improved inhibitory control performance in the control group relative to the $\mathrm{KC}$ group.

\section{School grades}

Independent $t$-tests revealed group differences on end of year school grades (Fig. 2). The KC group earned higher grades than the control group in Approaches to Learning $(t[57]=2.05, p$ $=.045, d=0.54)$, Health and Physical Development $(t[57]=2.23, p=.030, d=0.56)$ and Social and Emotional Development $(t[57]=3.69, p<.001, d=0.97)$. There were not differences between groups on Cognition and General Knowledge or Language 
Development and Communication $(p>.10, d \mathrm{~s}=-0.30$ and 0.09 for Cognition and Language respectively).

\section{Moderators of Treatment Effect}

Moderators of the treatment effect were tested with ANOVA, using change in social competence total score as the outcome variable. In these models, baseline levels of social competence (total score: $F[2,62]=5.45, p=.023$ and prosocial behavior subscale: $F[2,62]$ $=8.36, p=.005)$, cognitive flexibility (DCCS all trials: $F[2,55]=7.25, p=.009)$ and inhibitory control (Flanker: $F[2,55]=14.72, p<.001$; Fig. 3 ) significantly interacted with group to predict change in social competence. No other significant interactions were found $(p>.10)$. Children in the KC group with lower levels of social competence and executive functioning at baseline showed larger improvements in social competence over time relative to the control group.

\section{Intraclass correlation coefficients}

Intraclass correlation coefficients (ICCs) were computed to index the amount of variance in outcomes attributable to classrooms (see Appendix Table 2). As expected, ICCs were larger for teacher-reported outcomes (ICCs $=.19$ to .35 for TSC baseline and difference scores, ICCs $=.17$ to .48 for end-of-year grades). ICCs were close to zero (ICC < .02) for most behavioral tasks (baseline and difference scores) with the exception of the sharing task at baseline (ICC $=.10$ for self stickers), inhibitory control at baseline (ICC $=.23$ for Flanker), and change in delay (ICC $=.05$ for one vs. three trial types). Although there was a range of ICCs, classroom effects alone do not explain the pattern of results. However, the sizeable ICCs for some teacher-rated outcomes highlights potential limitations of relying on teacherbased measures when conducting research in schools. This accentuates the need for including larger numbers of teachers and classrooms in designs that can adequately model informant response biases in future work.

\section{Discussion}

This project demonstrated the feasibility and potential benefits of implementing a mindfulness-based prosocial skills training curriculum in a real world classroom setting. Results indicated improvements in response to intervention across a range of outcomes. Between-group analyses indicated that students who participated in the Kindness Curriculum (KC) training showed larger gains in teacher-reported social competence as compared to the control group. In addition, the control group acted more selfishly (sharing fewer resources with others) over time as compared to the $\mathrm{KC}$ group. Comparison of end of year school records showed higher marks for children in the intervention as compared to control group on indicators of learning, social-emotional development, and health. Notably, these differences emerged for second semester report card grades assigned approximately 3 months after the end of the intervention. Examination of effect sizes favored the KC group on measures of cognitive flexibility, specifically post-switch trials, and delay of gratification. Both groups showed improvement on the Flanker task, with a larger effect size observed in the control group, indicating that the intervention did not selectively improve inhibitory control. Overall, however, inspection of effect sizes lends support to a general 
pattern of change favoring the intervention group, even though not all outcomes were significant in the omnibus test of the group by time interaction, which highlights the need for larger samples in future studies.

Understanding differences in how individuals respond to intervention is useful, as there may be moderators of the intervention effect. Given the emphasis on prosocial skills development of this mindfulness-based curriculum, we focused on social competence as an outcome of interest potentially moderated by individual differences. It appears that the $\mathrm{KC}$ intervention may have been particularly beneficial for some children, specifically those with lower baseline functioning. Children in the intervention group who started out with lower social competence and lower executive functioning (indexed by inhibitory control and cognitive flexibility) at baseline showed greater improvements in social competence relative to the control group. This pattern of differential treatment effects is consistent with previous research documenting larger gains among children with poorer baseline function (Diamond \& Lee, 2011; Bierman et al., 2008). Taken together, the findings from this study suggest that there are benefits for a general education classroom and that children with deficits in executive function and social competence may experience additional gains.

That these changes were observed after a relatively short intervention period with a very modest dose of the intervention supports the practicality of this approach. The training shows promise as an accessible and cost-effective strategy to promote well-being and prosocial behavior by training non-cognitive skills that are also important for academic success. Taking a universal preventive approach may set children on a positive trajectory for ongoing development. As effects of self-control are found to follow a gradient, an intervention that produces even small increases in self-control could yield societal benefits by shifting the distribution of associated outcomes (Moffitt et al., 2011).

While teachers were not blind to study condition, the present methods include objective behavioral measures along with informant report. Additionally, report card grades were assigned approximately three months after the end of the intervention and grades were not higher across all subject areas for the intervention group. This suggests that teachers did not demonstrate a systematic bias in assigning student grades. Rather, differences may reflect particular domains of performance in which intervention group children demonstrated greater competence. The findings that individual differences moderated improvement in social competence further suggest a lack of uniform bias displayed by teachers in their ratings of children in the intervention group. The current study is limited by the relatively small sample size. Larger samples that enable analyses to account for the nested structure of data and follow-up to assess longer-term effects of training at 6 months and beyond are needed to more rigorously assess the effects of training. Assessment would be augmented by incorporating other objective measures such as cortisol (as a biological marker of stress) and third party observations of classroom behavior. Future work should also explore adding a parent component to training as recent research indicates stronger impact of child training when combined with parent training (Neville et al., 2013).

This study demonstrates the promise of incorporating mindfulness-based training into an early education curriculum. Overall, these results suggest that a relatively brief mindfulness- 
based training can enhance a range of academic and prosocial outcomes in young children. Clearly, more work is needed on the cultivation of kindness and compassion in young children and their parents and teachers. Training these skills and capacities early in life has important consequences throughout development, at both an individual and societal level.

\section{Supplementary Material}

Refer to Web version on PubMed Central for supplementary material.

\section{Acknowledgements}

We are grateful to all of the students and parents who participated in this project and the Madison Metropolitan School District for making this collaboration possible; Sharon Salzberg for her inspiration and feedback on the Kindness Curriculum; Lionel Newman and David Findley for their skillful project coordination; and undergraduate RAs for data collection. This research was generously supported by funding from the Caritas Foundation, Mental Insight Foundation, Mind \& Life Institute, Cremer Foundation, Baumann Foundation, Drs. Fran and Tim Orrok, Edward L. and Judith Steinberg, Chade-Meng Tan, Peggy Hedberg, and Arlene and Keith Bronstein. This project was also made possible in part by an NICHD core grant to the Waisman Center (P30 HD003352) and support from the Templeton Foundation and Fetzer Institute.

\section{References}

Anderson P. Assessment and development of executive function (EF) during childhood. Child Neuropsychology. 2002; 8(2):71-82. http://dx.doi.org/10.1076/chin.8.2.71.8724. [PubMed: 12638061]

Barkley RA. The executive functions and self-regulation: An evolutionary neuropsychological perspective. Neuropsychology Review. 2001; 11:1-29. http://dx.doi.org/10.1023/a:1009085417776. [PubMed: 11392560]

Becker B. Synthesizing standardized mean-change measures. British Journal of Mathematical and Statistical Psychology. 1988; 41:257-278. http://dx.doi.org/10.1111/j.2044-8317.1988.tb00901.x.

Bierman KL, Nix RL, Greenberg MT, Blair C, Domitrovich CE. Executive functions and school readiness intervention: Impact, moderation, and meditation in the Head Start REDI program. Development and Psychopathology. 2008; 20:821-843. http://dx.doi.org/10.1017/ s0954579408000394. [PubMed: 18606033]

Black DS, Milam J, Sussman S. Sitting-meditation interventions among youth: A review of treatment efficacy. Pediatrics. 2009; 124:e532-e541. http://dx.doi.org/10.1542/peds.2008-3434. [PubMed: 19706568]

Blair C. School readiness: Integrating cognition and emotion in a neurobiological conceptualization of children's functioning at school entry. American Psychologist. 2002; 57(2):111-127. http:// dx.doi.org/10.1037//0003-066x.57.2.111. [PubMed: 11899554]

Blair C, Razza RP. Relating effortful control, executive function, and false belief understanding to emerging math and literacy ability in kindergarten. Child Development. 2007; 78(2):647-663. http://dx.doi.org/10.1111/j.1467-8624.2007.01019.x. [PubMed: 17381795]

Cohen, J. Statistical power analysis for the behavioral sciences. 2nd ed.. Erlbaum: Hillsdale, NJ; 1988. http://dx.doi.org/10.4324/9780203771587

Conduct Problems Prevention Research Group. Teacher - Social Competence Scale. 1995. Retrieved from the Fast Track Project Web site, http://www.fasttrackproject.org

Davidson RJ, Dunne J, Eccles JS, Engle A, Greenberg M, Jennings P, Vago D. Contemplative practices and mental training: Prospects for American education. Child Development Perspectives. 2012; 6(2):146-153. http://dx.doi.org/10.1111/j.1750-8606.2012.00240.x. [PubMed: 22905038]

Davidson RJ, McEwen BS. Social influences on neuroplasticity: Stress and interventions to promote well-being. Nature Neuroscience. 2012; 15(5):689-695. http://dx.doi.org/10.1038/nn.3093.

Diamond, A. Normal development of prefrontal cortex from birth to young adulthood: Cognitive functions, anatomy, and biochemistry. In: Stuss, DT.; Knight, RT., editors. Principles of frontal 
lobe function. London, UK: Oxford University Press; 2002. p. 466-503.http://dx.doi.org/10.1093/ acprof:oso/9780195134971.003.0029

Diamond A, Lee K. Interventions shown to aid executive function development in children 4 to 12 years old. Science. 2011; 333(6045):959-964. http://dx.doi.org/10.1126/science.1204529. [PubMed: 21852486]

Eigsti I, Zayas V, Mischel W, Shoda Y, Ayduk O, Dadlani MB, Casey BJ. Predicting cognitive control from preschool to late adolescence and young adulthood. Psychological Science. 2006; 17(6):478484. http://dx.doi.org/10.1111/j.1467-9280.2006.01732.x. [PubMed: 16771797]

Eisenberg, N.; Spinrad, T.; Smith, C. The regulation of emotion. Mahwah, NJ US: Lawrence Erlbaum Associates Publishers; 2004. Emotion-related regulation: Its conceptualization, relations to social functioning, and socialization; p. 277-306.

Flook L, Smalley SL, Kitil MJ, Galla BM, Kaiser-Greenland S, Locke J, Kasari C. Effects of mindful awareness practices on executive functions in elementary school children. Journal of Applied School Psychology. 2010; 26(1):70-95. http://dx.doi.org/10.1080/15377900903379125.

Garon N, Bryson SE, Smith IM. Executive function in preschoolers: A review using an integrative framework. Psychological Bulletin. 2008; 134(1):31-60. http://dx.doi.org/ 10.1037/0033-2909.134.1.31. [PubMed: 18193994]

Greenberg MT, Weissberg RP, O’Brien MU, Zins JE, Fredericks L, Resnik H, Elias MJ. Enhancing school-based prevention and youth development through coordinated social, emotional, and academic learning. American Psychologist. 2003; 58(6-7):466-474. http://dx.doi.org/ 10.1037/0003-066X.58.6-7.466. [PubMed: 12971193]

Heckman JJ. The economics of inequality: The value of early childhood education. American Educator. 2011; 35(1):31-35. 47. Retrieved from ERIC database.

Lutz A, Brefczynski-Lewis JA, Johnston T, Davidson RJ. Regulation of the neural circuitry of emotion by compassion meditation: Effects of meditative expertise. PLoS ONE. 2008; 3(3):e1897. 26 http://dx.doi.org/10.1371/journal.pone.0001897. [PubMed: 18365029]

Lutz A, Slagter HA, Dunne JD, Davidson RJ. Meditation and the regulation of attention and emotion. Trends in Cognitive Sciences. 2008; 12(4):163-169. http://dx.doi.org/10.1016/j.tics.2008.01.005. [PubMed: 18329323]

MacLean KA, Ferrer E, Aichele SR, Bridwell DA, Zanesco AP, Jacobs TL, Saron CD. Intensive meditation training improves perceptual discrimination and sustained attention. Psychological Science. 2010; 21:829-839. http://dx.doi.org/10.1177/0956797610371339. [PubMed: 20483826]

McCloskey, G.; Perkins, L.; Van Divner, B. Assessment and intervention for executive function difficulties. New York, NY US: Routledge/Taylor \& Francis Group; 2009.

Mischel W, Shoda Y, Peake PK. The nature of adolescent competencies predicted by preschool delay of gratification. Journal of Personality and Social Psychology. 1988; 54(4):687-696. http:// dx.doi.org/10.1037/0022-3514.54.4.687. [PubMed: 3367285]

Mischel W, Shoda Y, Rodriguez ML. Delay of gratification in children. Science. 1989; 244(4907): 933-938. http://dx.doi.org/10.1126/science.2658056. [PubMed: 2658056]

Miyake A, Friedman NP, Emerson MJ, Witzki AH, Howerter A, Wager TD. The unity and diversity of executive functions and their contributions to complex "frontal lobe" tasks: A latent variable analysis. Cognitive Psychology. 2000; 41:49-100. http://dx.doi.org/10.1006/cogp.1999.0734. [PubMed: 10945922]

Moffitt TE, Arseneault L, Belsky D, Dickson N, Hancox RJ, Harrington H, Caspi A. A gradient of childhood self-control predicts health, wealth, and public safety. Proceedings of the National Academy of Sciences. 2011; 108(7):2693-2698. http://dx.doi.org/10.1073/pnas.1010076108.

Napoli M, Krech PR, Holley LC. Mindfulness Training for Elementary School Students: The Attention Academy. Journal of Applied School Psychology. 2005; 21(1):99-125. http://dx.doi.org/10.1300/ J370v21n01_05.

Neville HJ, Stevens C, Pakulak E, Bell TA, Fanning J, Klein S, Isbell E. Family-based training program improves brain function, cognition, and behavior in lower socioeconomic status preschoolers. Proceedings of the National Academy of Sciences. 2013; 110(29):12138-12143. http://dx.doi.org/10.1073/pnas.1304437110. 
Prencipe A, Zelazo P. Development of affective decision making for self and other. Psychological Science. 2005; 16(7):501-505. http://dx.doi.org/10.1111/j.0956-7976.2005.01564.x. [PubMed: 16008779]

Raver CC. Low-income children's self-regulation in the classroom: Scientific inquiry for social change. American Psychologist. 2012; 67(8):681-689. http://dx.doi.org/10.1037/a0030085. [PubMed: 23163459]

Snijders, TA.; Bosker, RJ. Multilevel analysis: An introduction to basic and advance multilevel modeling. 2nd ed.. London: Sage; 2012.

Shoda Y, Mischel W, Peake PK. Predicting adolescent cognitive and self-regulatory competencies from preschool delay of gratification: Identifying diagnostic conditions. Developmental Psychology. 1990; 26(6):978-986. http://dx.doi.org/10.1037/0012-1649.26.6.978.

Spinrad T, Eisenberg N, Cumberland A, Fabes R, Valiente C, Shepard S, Guthrie IK. Relation of emotion-related regulation to children's social competence: A longitudinal study. Emotion. 2006; 6(3):498-510. http://dx.doi.org/10.1037/1528-3542.6.3.498. [PubMed: 16938090]

Weng HY, Fox AS, Shackman AJ, Stodola DE, Caldwell JZK, Olson MC, Rogers GM, Davidson RJ. Compassion training alters altruism and neural responses to suffering. Psychological Science. 2013; 24(7) http://dx.doi.org/10.1177/0956797612469537.

Zelazo PD, Anderson JE, Richler J, Wallner-Allen K, Beaumont JL, Weintraub S. II. NIH TOOLBOX COGNITION BATTERY (CB): MEASURING EXECUTIVE FUNCTION AND ATTENTION. Monographs of the Society for Research in Child Development. 2013; 78:16-33. [PubMed: 23952200]

Zelazo, P.; Carlson, S.; Kesek, A. Handbook of developmental cognitive neuroscience. 2nd ed.. Cambridge, MA US: MIT Press; 2008. The development of executive function in childhood; $p$. 553-574. 


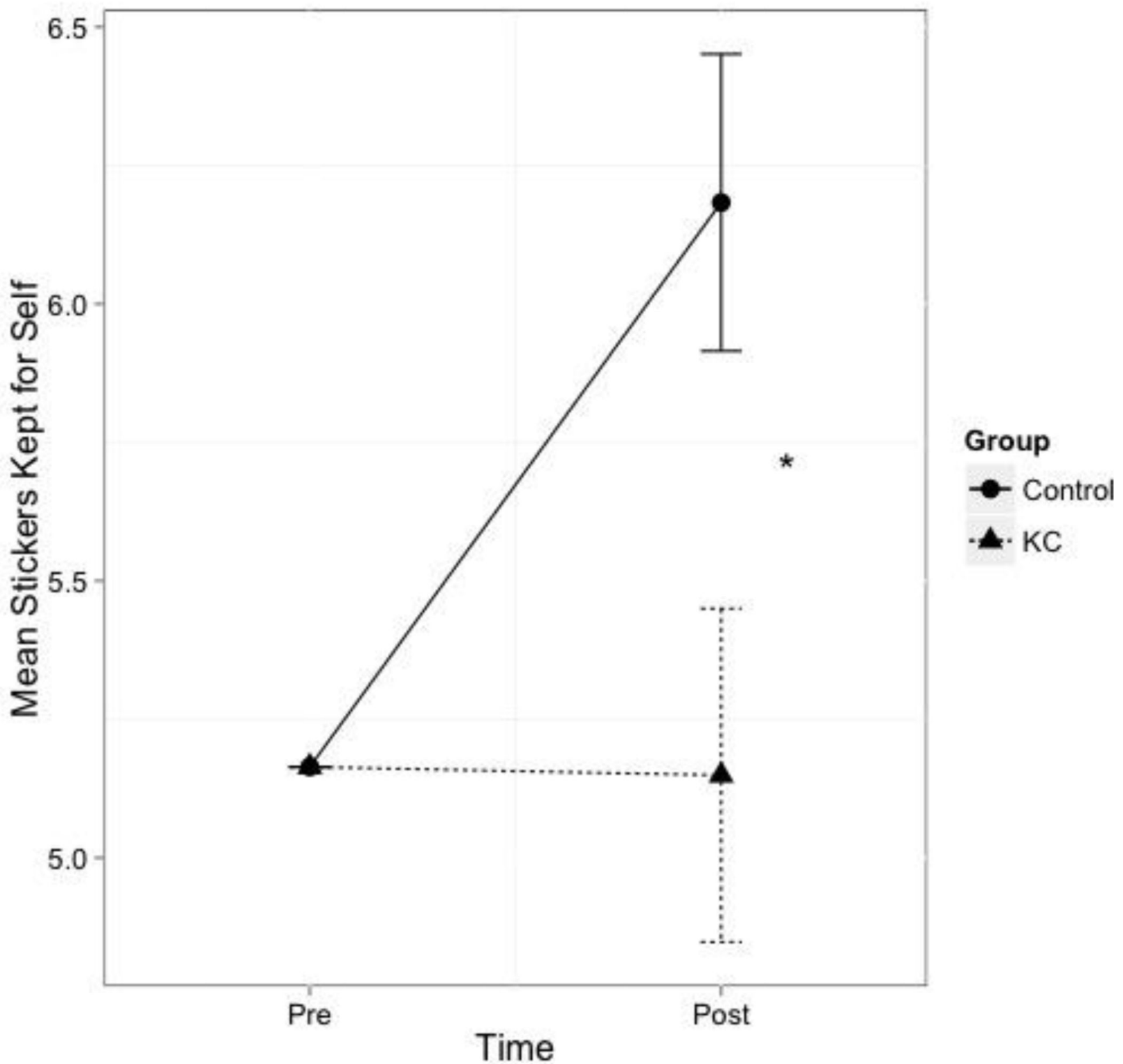

Figure 1.

Treatment effects on sharing behavior. Over time the control group kept more stickers for themselves (shared less with others) on average across all trials relative to $\mathrm{KC}$ group. Standard error bars displayed. Plot displays results of RMANOVA analysis controlling for sharing at baseline. 


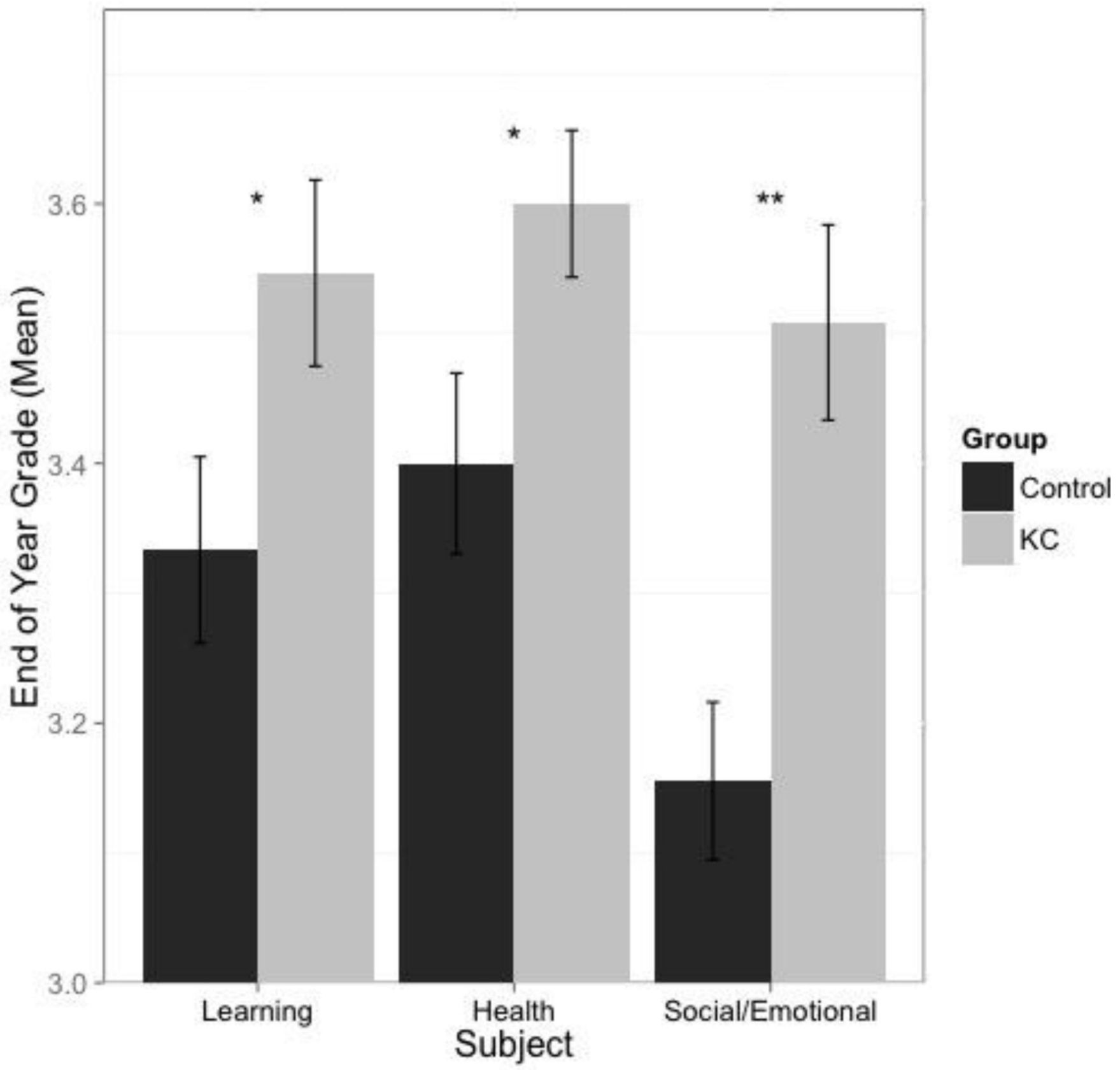

Figure 2.

Group differences in end of year school grades. Kindness Curriculum group earned higher marks than control group across three subject areas. 


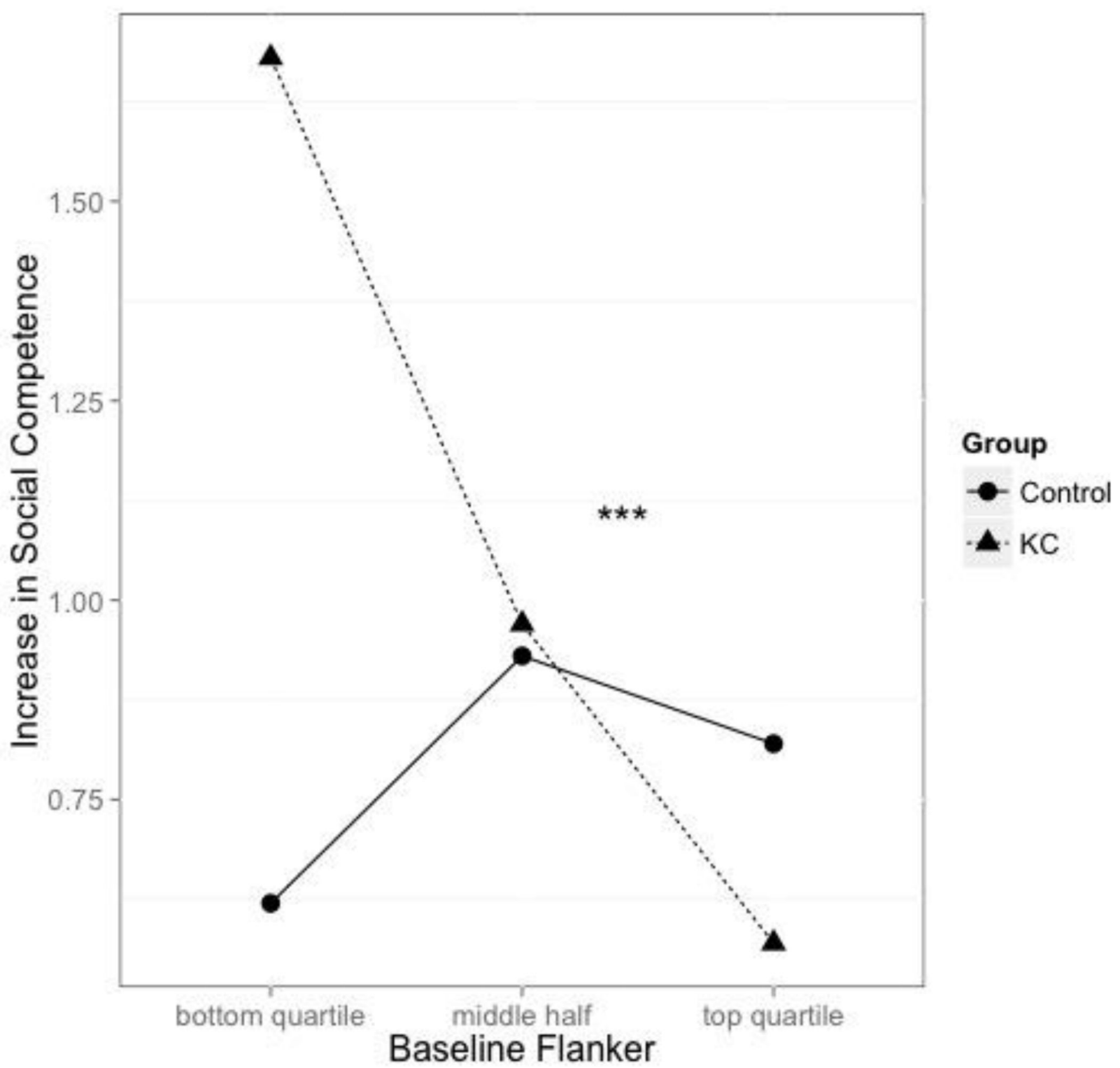

Figure 3.

Individual differences in baseline inhibitory control predict response to intervention. Baseline inhibitory control (Flanker) predicts change (post - pre) in social competence. Mean change in social competence is displayed separately for $\mathrm{KC}$ and control group for bottom quartile, middle half, and top quartile performances on Flanker at baseline (quartiles computed for display purposes only). Children with lower inhibitory control in the KC group increased more in social competence compared to the control group. Interaction significant at $p<.001$ 
Flook et al.

Page 16

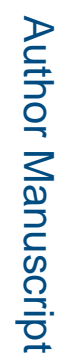

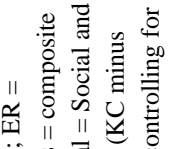

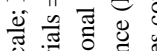

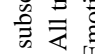

的

贾 议

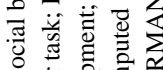

웅 홍

II

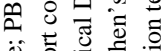

(5)

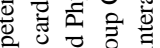

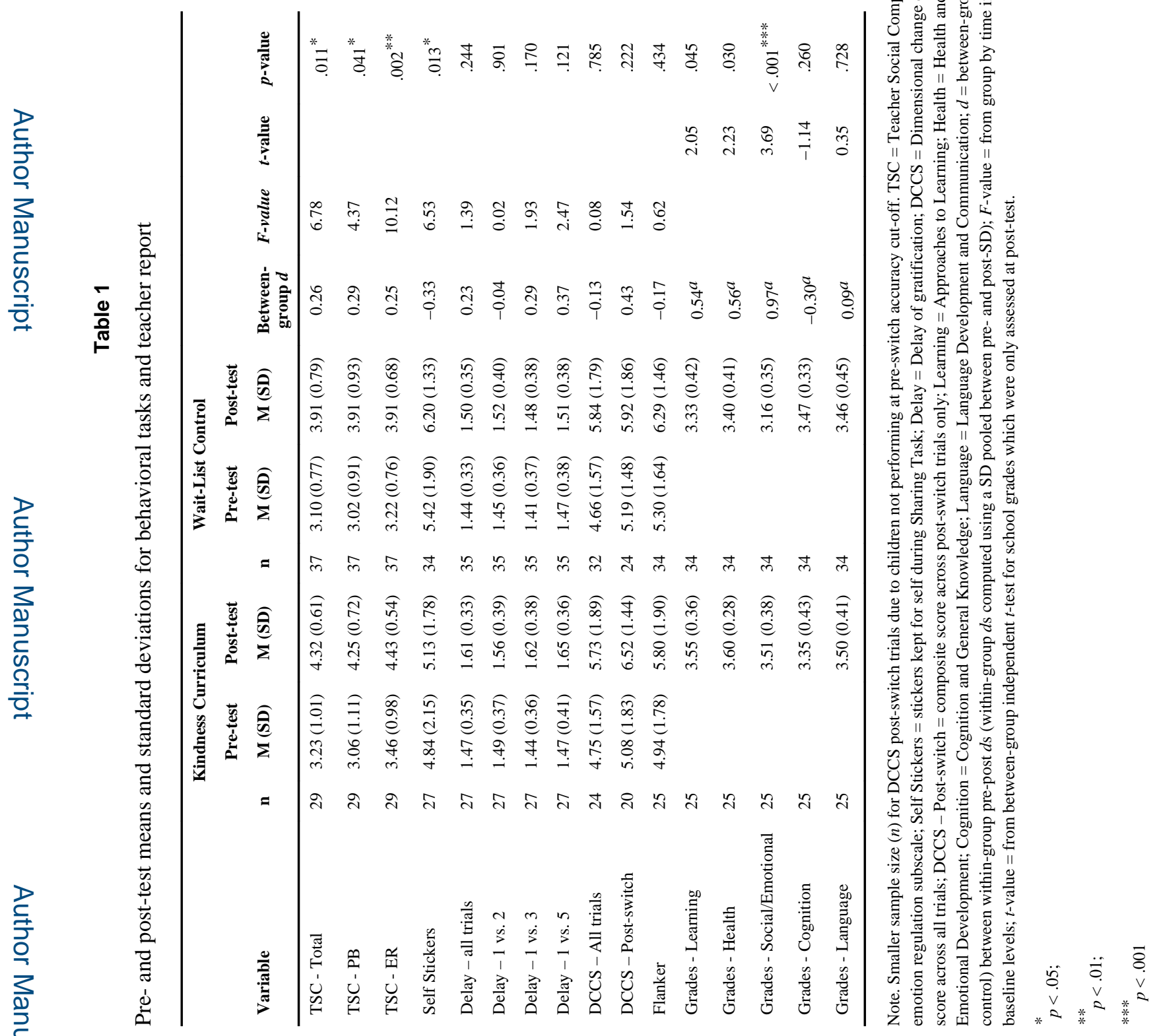

Dev Psychol. Author manuscript; available in PMC 2015 June 28. 


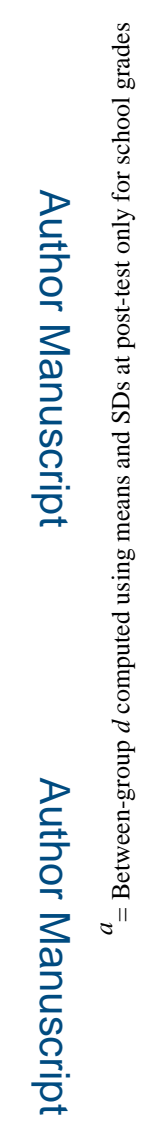

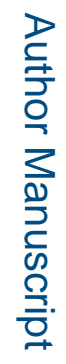

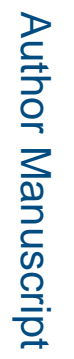

Dev Psychol. Author manuscript; available in PMC 2015 June 28. 